\title{
Entrelacs
}

Cinéma et audiovisuel

\section{Fluidité et plasticité dans les Nuits fauves de Cyril Collard}

\section{Thierry Giaccardi}

\section{(2) OpenEdition}

\section{Journals}

\section{Édition électronique}

URL : http://journals.openedition.org/entrelacs/359

DOI : 10.4000/entrelacs.359

ISSN : 2261-5482

Éditeur

Éditions Téraèdre

Référence électronique

Thierry Giaccardi, «Fluidité et plasticité dans les Nuits fauves de Cyril Collard », Entrelacs [En ligne],

9 | 2012, mis en ligne le 22 octobre 2012, consulté le 19 avril 2019. URL : http://

journals.openedition.org/entrelacs/359; DOI : 10.4000/entrelacs.359

Ce document a été généré automatiquement le 19 avril 2019.

Tous droits réservés 


\title{
Fluidité et plasticité dans les Nuits fauves de Cyril Collard
}

\author{
Thierry Giaccardi
}

1 Les toutes premières scènes des Nuits fauves ${ }^{1}$ se déroulent au Maroc. L'importance de ce lieu inaugurant la fiction ou plutôt l'autofiction - cas assez rare au cinéma - ne doit pas être ignorée: le personnage déambule dans les rues d'une ville marocaine avec une frivolité et une complaisance pouvant agacer à première vue. Or, Jean confie, en voix off, qu'il a l'impression « de traverser la vie comme ces touristes américains traversent les pays visités, pour faire le plus de villes possible. » Et il ajoute : « Les natifs du Sagittaire veulent toujours être ailleurs que là où ils sont. » Cette manière de traverser, d'habiter un lieu définit aussi, sans doute, celle d'habiter son propre corps.

2 Ainsi, par ces paroles en apparence anodines, Cyril Collard/Jean affirme une des grandes vérités foisonnantes, baroques, doubles, des Nuits fauves, formant à la fois le soubassement idéologique $\mathrm{du}$ film et son imaginaire, féérique, onirique, voire astrologique, en tout cas un imaginaire propre au conte davantage qu'à celui de la chronique, plus sec, comme le sujet du film semblait y inviter. D'une part, le monde dans toute son exubérance peut être dépaysant. L'homme s'y sent parfois comme un touriste ; il n'appartient pas totalement à cette réalité soit dure (celle des nouvelles cités, comme dans la scène où Jean effectue un repérage avec Kader pour une publicité), soit généreuse (les scènes au bord de mer ou dans cette Afrique du nord pasolinienne). Et, d'autre part, le réel extérieur semble irréel, poreux, tels l'individu qui n'est pas "éternel $»^{2}$ ou les réalisations humaines ${ }^{3}$ comme cette ville marocaine faite de briques de terre apparaissant en haut d'une colline à l'instar d'une ville de contes et légendes. Ces paysages naturels ou urbains ne semblent renvoyer qu'à une fonction : celle d'émerveiller, ou plutôt celle de témoigner d'un certain accomplissement, on pourrait ajouter d'une certaine majesté de la profusion de la nature et des productions humaines. L'homme collardien est, fondamentalement, un homo faber au sens où il construit et se construit, intérieurement. Or, L'homo faber, mais aussi l'homosexuel, sont présentés dans la fiction comme agnostiques : pour eux, il n'y a aucune fatalité (biologique voire épidémiologique). Ils ne sont pas les gardiens d'une révélation divine impliquant un monde figé mais bien ceux 
d'une révolution humaine permanente : la transformation du monde, de soi. La sexualité dans Les Nuits fauves découle de cette vision des choses et des êtres, monde de la plasticité que l'univers du conte saisit bien, peut-être mieux que les nouvelles théories de l'homme insistant sur l'arbitraire, le construit, mais d'une manière trop sèche, ne laissant que rarement sa part au rêve. Cette vision repose sur le refus de choisir comme l'indique Jean à Laura. Elle n'implique pas l'idée d'une « altération du moi » freudienne, ce qui est sans doute le grand tour de force du film : les maladies mentales ne sont pas sollicitées afin de justifier ceci ou cela. La vie prométhéenne reprend ses droits, et du coup, le mythe reprend sa place.

3 Il est vrai que la sexualité dans Les Nuits fauves semble recevoir un sens relativement restreint à première vue : l'accouplement au sens de coït vaginal ou anal. Elle pourrait ainsi apparaitre, paradoxalement, en deçà, seconde, par rapport à l'expérience médiate de la vie et des grands transports qui bouleversent l'individu. Le discours tendrait à lui ôter le caractère fondateur d'une appréhension du monde (dans les deux sens de l'expression). Cependant, si Jean jouit d'être sodomisé par Samy ne pense-t-il pas au même moment à Laura et à l'enfant qu'ils pourraient concevoir ensemble ? Le montage du film indique nettement que c'est bien le cas : dès le début du film les scènes avec Jean et Laura et Jean et Samy se succèdent avec une grande rigueur. Or, le montage dans Les Nuits fauves exprime plusieurs idées fondamentales pouvant apparaître comme contradictoires, alors que celles-ci relèvent de différents plans, de différentes logiques narratives, intellectuelles et spirituelles. Certes, il exprime la dualité, parfois la duplicité, des choses et des êtres, mais il suggère dans le même temps que celles-ci peuvent se résorber à des niveaux de conscience particuliers. Ne pourrait-il s'agir alors que de cette «opposition toute classique » dont parle Rohmer, et qui serait la grande affaire du cinéma, c'est-à-dire «l'opposition de deux ordres, l'un naturel, l'autre humain, l'un matériel, l'autre spirituel, l'un mécanique, l'autre libre, l'un celui du désir de l'appétit, l'autre de l'héroïsme ou de la grâce » ${ }^{4}$ ?

$4 \quad$ Les Nuits fauves illustrent cette idée très concrète qu'être pénétré par un pénis et pénétrer avec le sien un autre corps renvoie plus subtilement à l'unicité, davantage sans doute qu'à l'unique. Voire à une union cosmique. La scène entre Samy, Jean et Sylvie, une jeune étudiante en histoire de l'art, l'exprime d'une manière très plastique (la référence à la peinture est explicite dans le dialogue et le lieu). Filmée en plongée, dans la bibliothèque où Dali et Gala se sont rencontrés, les trois sont brièvement mais intimement enlacés sur un canapé: ils composent une figure de l'entremêlement, auquel fera écho, après la première rupture avec Laura, une scène où un groupe d'homosexuels font l'amour sous un pont. Dans cette dernière scène, l'œil de la caméra, loin de donner lieu à un « regard clinique ", s'emploie à restituer une chorégraphie des corps s'ébattant à l'unisson et fait apparaître une sorte de vie symbiotique grâce, notamment, à ces silhouettes autour de Jean. On pense à la différence fertile dont parle Metz entre « fins expressives » et « étude de détail » à propos de l'utilisation du gros plan ${ }^{5}$.

5 C'est que la sexualité, si elle fascine l'auteur des Nuits fauves dans ce qu'elle procure de jouissance à la vue et au toucher des organes sexuels (les portraits nus de Laura sont aussi explicites que magnifiques) mais aussi dans son caractère "morbide», ne peut agir comme une taxinomie réduisant l'individu à un hétérosexuel ou un homosexuel. Dans la scène où Samy se rend chez Jean pour la première fois, le court dialogue qui suit entre les deux est particulièrement révélateur. Samy lui demande : "Mais t'es qui au fait? " La réponse de Jean, "Je suis moi », ne le satisfait pas. Samy lui rétorque: «T'aimes les 
garçons? » Pour Samy, la sexualité définit l'individu, ce que réfute Jean ${ }^{6}$. Du reste, la relation de ce dernier avec Laura n'évoque pas tant le couple qu'une relation idéalisée. D'où le fait qu'il affirme se sentir " propre » lorsqu'il revient vers elle, ce qui confère à Laura un statut angélique, allant jusqu'à rendre possible, sur un certain plan, une rémission des péchés de Jean (« les pires trucs » dit-il à Marc). La référence biblique est explicitée par Marc citant saint Jean : «Pour les êtres purs, tout est pur». Collard se révèle, ici, l'héritier de la tradition gidienne, laquelle exprime la sexualité dans toute sa complexité mais aussi dans toute sa disponibilité, en utilisant paradoxalement un langage sacré la niant au profit de l'amour divin, pouvant à la fin conduire au renoncement de la chair (La Porte étroite). En fin de compte, cette relation entre Jean (nouveau saint Jean ?) et Laura contribue à brouiller l'identité de "pédé » du héros. Jean, en posant la question à Laura, « qu'est-ce que tu sais des pédés, toi ? ", la pose du même coup au spectateur.

La sexualité serait donc perçue comme une condition de la révolte de l'homme, possiblement contre l'absurdité du monde, contre une certaine mesquinerie de la vie sociale, et contre la maladie et sa représentation. Elle est, en tout cas, une voie et non une finalité : l'homme désire, jouit, mais ne s'abîme pas dans l'acte sexuel. Noria, la belle Marocaine énigmatique, hiératique, personnage intercesseur ${ }^{7}$ apparaissant dans les premières scènes, a bien saisi ce trait chez Jean : « Tu t'es révolté par le sexe parce que tu n'as rien trouvé d'autre. ${ }^{8}$ » Ainsi, le scandale ne serait plus dans l'acte définissant l'individu comme une certaine morale continue à le suggérer en posant ainsi une métaphysique forcément contraignante, mais dans le fait que l'individu peut choisir librement son acte sans être réduit à celui-ci, selon un polymorphisme revendiqué par Collard. Cette dernière notion rappelle celle du «corps pluriel» de Barthes, lequel n'hésitait pas à exprimer sa fascination pour « le corps socialisé, le corps mythologique, le corps artificiel $»^{9}$. Dans un beau travelling nocturne où Samy et Jean se promènent dans Pigalle, Jean raconte qu'il a fait le "tapin ", une fois. Samy lui demande s'il avait alors besoin d'argent, Jean se contente de répondre que non. Cette réponse ne manque pas de renvoyer à l'acte gratuit de type gidien et à son ambiguïté foncière : exprime-t-il la liberté ou l'absurdité de la vie?

7 D'une certaine manière, on peut affirmer que Les Nuits fauves sont un des rares films existentialistes de la fin des années 80 dans la mesure où la plasticité de la vie y est revendiquée comme une condition première de l'existence, même si elle peut se durcir au cours d'événements ultérieurs. Plasticité, du reste, qui a aussi sa face cachée et que la critique n'a pas manqué de relever et de critiquer, à juste titre: Jean ne prévient pas Laura qu'il est séropositif la première fois qu'ils font l'amour ${ }^{10}$.

8 Si l'univers de Collard peut donner l'illusion d'être fait à la fois de faux-semblant (les danses ou les chansons folkloriques, marocaines ou espagnoles) et d'une nature qui se donne sans compter, c'est donc qu'il repose sur un principe saisissant, celui de la dualité foncière de toute chose : non pas une dualité que l'intellect saisit et ressasse en cherchant à la dépasser (la dualité des philosophes), mais, au contraire, une dualité revendiquée avec une insouciance déconcertante, vécue jusqu'au bout, et qui finit par être résorbée. De sorte que le mythe de l'hermaphrodite résume fort bien les différentes tonalités du film. Une des définitions collardiennes possibles de l'hermaphrodite pourrait être cette phrase prononcée par Jean : «je suis fait de morceaux de moi-même éparpillés et recollés ensemble n'importe comment». L'autofiction de Collard se distingue précisément par cette utilisation du mythe de celle d'un Guibert, son contemporain, lui-même grand photographe, même si à la fin du film Collard est tenté 
par le cinéma-vérité en parlant de choses graves comme la rupture ou les stigmates de la maladie (les scènes dans la salle d'hôpital où le héros subit une opération de chirurgie dermatologique $\left.{ }^{11}\right)$.

9 Cette dualité semble être la condition du désir chez Collard. Cependant, si elle s'exprime en particulier - mais pas seulement - dans la sexualité de Jean, elle ne peut être vécue dans le même temps. Elle im/pose la succession, voire le mensonge, c'est-à-dire une mise en scène de la « liaison ", du couple éphémère et de ses objets insolites comme de ses rebuts : lorsque Jean est avec Samy, Laura est exclue, et réciproquement. Le montage alterné, et les notions de cadre et de hors-champ spécifiques qui en résultent, exprime aussi cette vérité au point que, sur un certain plan, on pourrait parler de dissociation. A cet égard, les courtes scènes montées en coupe franche où Jean conduit successivement Samy et Laura sous un tunnel sont particulièrement efficaces. Une autre scène, très bergmanienne, est exemplaire à la fois de la mise en scène de Collard et des types de relation qu'établit le héros avec les êtres aimés : en prenant leur petit déjeuner, Jean et Laura se félicitent de vivre une vie de couple sans heurts depuis que Laura est venue habiter chez Jean. Or, le plan, fortement divisé verticalement en deux, semble contredire le dialogue, avec, à gauche, la table du petit déjeuner, et à droite un miroir réfléchissant le salon où se trouvent une table basse et un téléphone. Jean va d'une pièce, c'est-à-dire d'une partie du plan, à l'autre, afin de répondre à un appel téléphonique qu'on devine de Samy. Il est vrai que Laura finira par imposer sa présence dans la relation entre Jean et Samy : Jean parlera alors de « possession » et non d'amour car il ne peut pas envisager la monogamie ${ }^{12}$, ce qui reviendrait à nier sa nature bisexuelle. Mais cette intrusion (dans le plan) ne pourra que donner lieu à une confrontation particulièrement violente et aboutir à la fin de la relation avec Jean.

10 On peut voir initialement Les Nuits fauves comme un film de l'immaturité, un film de l'adolescence comme il y a des romans de l'adolescence où le personnage devine, ou plutôt entraperçoit des vérités mais semble condamner à une certaine immobilité des sentiments (Le Grand Meaulnes), provoquée en partie par le refus de choisir (de se choisir). Ainsi, ces plans de voiture décapotable, au début du film, où Jean, cheveux au vent, conduit avec une grande insouciance dans les rues de Paris. Or, ces plans, même s'ils ne sont pas dénués de narcissisme, ne sont pas gratuits : ils contiennent une charge érotique certaine visant à créer une "icône » fascinant les deux sexes. Ce sont des images nécessaires à ce point du récit précisément parce que le héros, par la suite, assumera ouvertement son côté passif dans sa relation homosexuelle avec Samy ${ }^{13}$. Mais, on peut aussi comprendre Les Nuits fauves comme un film réalisé par un homme dont la maladie incurable l'a convaincu que les choix et les codes sont arbitraires et révèlent davantage une légèreté de l'être qu'un sérieux ${ }^{14}$ ou un ordonnancement renvoyant à de grands principes métaphysiques. Les frontières entre la vie et le rêve, à l'instar de celle entre l'homme et la femme, s'estompent, sans, toutefois tomber dans l'informel, l'abstraction ou l'univers panique.

11 De plus, Les Nuits fauves doivent être vues comme un film de la nuit mystique, du doute métaphysique saisissant l'homme à la fin de sa vie, mais surtout des puissances qui l'assaillent dans la mesure où un jeune homme ne peut se décider vraiment à accepter sa mort (voir les scènes poignantes où Jean pleure dans les bras de Samy et de son ancienne maitresse). En effet, «l'éphèbe" ne doit pas mourir ou alors il doit être sacrifié. Le personnage de Jean voit le monde différemment que l'homme pris dans les soucis de la vie quotidienne. On peut même aller jusqu'à parler d'un plan de la vibration (les 
premières scènes où Jean conduit sa voiture à toute allure et les dernières où l'image des paysages perd de sa netteté donnant ainsi à voir "le grain de l'image »), voire du déchirement du voile car l'arrière-plan astrologique, alchimique, n'est jamais loin : Jean porte avec ostentation une croix de vie et une clé évoquant la clé des songes, Samy parle de purification avant l'œuvre au noir. Et, en ce sens, Les Nuits fauves peuvent renvoyer, consciemment ou non, à La Nuit de la Walpurgis de Meyrink dont le héros, condamné à mourir à cause d'une maladie incurable comme Jean, se rebelle ${ }^{15}$.

Enfin, et peut-être surtout, le film se situe dans la tradition inaugurée par Pasolini, celleci n'étant pas toujours reçue sans malentendu car elle n'hésite pas à traiter de la religion : c'est la tradition du personnage homosexuel ou bisexuel, porteur de vérités fondamentales, annonciateur pourrait-on dire aussi (Théorème), aussi à son aise dans le monde archaïque, plus peut-être, que dans le monde moderne ${ }^{16}$. C'est ainsi que dans Les Nuits fauves la ville renvoie davantage à la nuit, à une certaine fatigue, à la maladie peutêtre, à un fort érotisme aussi, alors que les paysages naturels sont baignés dans une très belle clarté, expriment un état d'innocence auquel Jean semble vouloir aspirer plus qu'à tout autre chose, et insufflent au héros une nouvelle vigueur, comme dans le dernier plan du film où Jean/Cyril Collard accède à une sorte d'immortalité par le cri dans un paysage désertique. Le corps de l'homme est divinisé, comme chez Pasolini. Il se joue même de la maladie en se modelant sur la statuaire gréco-romaine mais aussi, dans la dernière séquence, sur la statue du Christ pantocrator, alors que, dans le même temps, les dialogues laissent deviner une certaine angoisse devant la mort. Le héros peut enfin entrer en sympathie totale avec les choses et les êtres de ce monde.

De ce point de vue, rappelons que Jean apparaît très vite comme un héros solaire ce qui contribue fortement à donner une tonalité apollinienne au film : dans une des premières scènes des Nuits fauves, après une nuit agitée, Jean se dirige vers le balcon de son appartement, torse nu, alors que le soleil se lève, et, dans la scène suivante, Kader le présente comme son chef opérateur et se croit obliger d'expliquer à Laura que " c'est la personne qui fait la lumière, le cadre ». Mais c'est sans doute la courte scène après l'aveu de sa maladie - où Jean est à nouveau sur son balcon et regarde le lever du soleil - qui donne lieu au plus beau champ-contre-champ du film: le personnage est filmé en plan rapproché, son buste se réfléchit sur la baie vitrée. Ce plan - émouvant autoportrait de l'artiste au seuil de la mort - est suivi d'un plan en plongée de Paris et du soleil se levant en bas à droite de l'écran, puis d'un zoom sur le soleil venant occuper le point central de l'image : l'écran devient jaune, cette " couleur de l'éternité » qui « est la plus chaude, la plus expansive, la plus ardente des couleurs, difficile à éteindre, et qui déborde toujours les cadres où l'on voudrait l'enserrer. ${ }^{17}$ Enfin, dans l'un des tout derniers plans du film, alors qu'il se retrouve au bout de l'Europe (du monde?), le soleil se superpose sur la tête de Jean avant de se coucher et de se lever à nouveau dans un mouvement accéléré de l'image, avec, en contre-champ, en plan rapproché, le visage christique de Collard et, en voix off, son monologue intérieur affirmant qu'il est « vivant ».

Les œuvres crépusculaires sont toujours nostalgiques du lever du soleil; certaines, refusant de s'absorber dans le néant proche, témoignent que la vie continue et semblent pencher en faveur du cycle ouvert davantage que de la biographie fermée, aussi bouleversante soit-elle. Dans la dernière scène du film, Jean déclare : «Je suis vivant, le monde n'est pas seulement une chose posée là, extérieure à moi-même. J'y participe. Il m'est offert. Je vais peut-être mourir du sida mais ce n'est plus ma vie. Je suis dans la vie. » Cette déclaration est suivie d'un mouvement d'appareil d'une rare beauté au cinéma 
donnant lieu à une scène où l'image exprime la vie à l'unisson avec le monde, intérieur et extérieur. L'écran tend une fois de plus vers la toile monochrome, un bleu strié par les reflets du soleil, atteignant cette limite dont parle Bonitzer à propos du gros plan : « La limite, la tentation et le grain de réel - ou de folie- du gros plan, c'est l'évanouissement de toute représentation. ${ }^{18}$ " Le refus du pathétique est la grande vérité des Nuits fauves : il est intimement lié à l'expression d'une grande liberté sexuelle de l'homme, et, derrière ou en dépit des convenances, d'une certaine continuité entre les civilisations débouchant sur une vision apaisée de la nature et des corps participant aux chants du monde.

\section{NOTES}

1. La poétique du film diffère sensiblement de celle du livre, au point qu'on peut affirmer que la visée esthétique et éthique change légèrement, peut-être à l'insu de Collard: il est tentant de suggérer que la version écrite serait plus gidienne et la version filmée plus pasolinienne.

2. Comme le dit avec une simplicité et un dévouement bouleversants l'infirmière s'occupant de Jean. Rappelons que le film, sorti en 1992, est l'adaptation par l'auteur de son livre paru en 1989, soit un an avant la parution du livre de Guibert, A l'ami qui ne m'a pas sauvé la vie, livres qui jouèrent un rôle important dans les attitudes françaises face à la maladie à la fin des années 80 . Le film de Collard est sorti la même année que le film de Kenneth Branagh, Peter's friends, mais un an avant celui de Jonathan Demme, Philadelphia. L'originalité avec laquelle Collard parle de l'homosexualité et du sida ne s'explique qu'en partie par sa propre maladie. C'est sa vision du monde, qu'on pourrait qualifier de gréco-romaine, qui fait de Collard un précurseur dans le cinéma français, place qu'on ne lui attribue que trop rarement. Ainsi, on notera qu'il n'y a aucune amertume chez lui, d'où un certain stoïcisme ; aucun tabou non plus ; mais une grande complicité avec les éléments, allant jusqu'à accepter la maladie. Alors que Pasolini est le cinéaste d'un monde archaïque mais aussi d'un monde panique, Collard est le cinéaste de l'acceptation héroïque de la permanence du monde.

3. Dans cette scène, il y a un mouvement des yeux de Collard vers la caméra non voulu, qui transforme en une fraction de seconde la fiction en documentaire. Cette fraction de seconde est un des moments les plus bouleversants de l'autofiction, écrite ou filmée.

4. ROHMER, Eric, Le Goût de la beauté (Paris : Flammarion, 1989), p. 93.

5. METZ, Christian, Essais sur la signification au cinéma, tome II (Paris : Klincksiek, 1972), p. 90.

6. A la fin du film Samy choisira de rejoindre un groupuscule d'activistes d'extrême-droite, ce qui exprime bien à la fois sa confusion et son attirance pour une virilité agressive.

7. Noria apparaît comme un personnage de conte, de rêve, mais n'en énonce pas moins des vérités fondamentales sur Jean. C'est elle qui lui conseille de tirer profit de «l'épreuve de la maladie ".

8. Dans la bouche de Noria, cette affirmation peut sembler réductrice et, somme toute, assez sévère (faux choix, pis aller, ou absence de choix). Une révolte sexuelle, c'est-à-dire une sexualité débridée, n'en est pas moins une révolte, n'est pas moins "révolte " qu'une révolte d'un autre type. En outre, il est possible d'affirmer que, chez Collard, elle vise à accéder à un plan métaphysique, qu'elle est aussi une discours sur Dieu ou son absence.

9. BARTHES, Roland, Le Plaisir du texte (Paris : Seuil, 1973), p. 39. 
10. Si cette conduite est impardonnable, elle n'est pas non plus dénuée d'une certaine logique selon le personnage : celle du rêve davantage que d'un refus plat des réalités avilissantes. Jean, qui a éprouvé le besoin de s'en confier à Marc, lui dit en effet que «c'était comme dans un rêve, comme si j'avais oublié que ce virus fait partie de moi ».

11. Ces scènes d'hôpital anticipent sur celles d'une autre autofiction célèbre, Caro diario de Nanni Moretti, de sorte qu'on peut se demander si elles ne constituent pas un sous-genre garantissant de manière forte l'aspect documentaire de la fiction.

12. Le film n'est pas dénué de nostalgie pour le couple monogame : après la violente altercation suivant la scène où Laura découvre Samy et l'ancienne amie de Jean dans l'appartement de ce dernier, un plan sur un bateau-mouche se focalise sur un couple de personnes âgées dansant sur le pont du bateau, suivi d'un très beau panoramique sur Jean et Laura enlacés sous un pont. Ici le montage dégage une très grande force, l'image discourant sur l'état du couple du point de vue de l'histoire et du point de vue subjectif du personnage.

13. C'est donc avec une certaine habileté dans le découpage que Collard fait suivre cette scène avec la scène où Jean va danser avec Samy en discothèque et dans laquelle Jean apparaît comme un personnage plus camp, pour citer Sontag, laquelle a popularisé cette notion. Voir Sontag Susan, Against Interpretation and Other Essays (New York: Farrer Strauss \& Giroux, 1964), en particulier son essai « Notes on Camps ». Collard joue ainsi sur les deux registres : affirmer que l'homosexuel peut être à la fois viril et efféminé. Sans nécessairement penser à La Cage aux folles, film loin d'être dénué d'intérêt, toute une tradition européenne et plus encore américaine, n'a pas hésité à mettre en avant le modèle, sans doute au détriment d'autres, selon lequel le fait d'être gay signifierait être efféminé. Le numéro de cabaret qui suit n'a pas de valeur dramatique en soi mais s'apparente davantage à la scène "manifeste ", exprimant un contenu fort; par ailleurs c'est une scène d'une grande poésie urbaine. Cette alternance de scènes homosexuelles et de scènes hétérosexuelles avec Laura, peut être qualifiée de systématique et, donc, être accusée de manquer de de finesse. Elle est nécessaire pour exprimer l'aspect frénétique, compulsif de la recherche de la vérité de l'Être chez le héros dans la mesure où Collard joue mezzo voce, avec une certaine retenue, ce qui donne une très grande force à sa composition (de soi) et laisse deviner le « rugissement » intérieur dont la chanson du générique parle. On peut aussi avancer l'idée qu'elle participe de l'esthétique de l'inventaire qui peut se comprendre dans une œuvre testamentaire.

14. Dans une scène révélatrice, Jean rit devant la peine de Laura qui lui reproche sa duplicité ou disons son infidélité, non parce qu'il est indifférent mais parce qu'il est déjà « ailleurs ».

15. Toutefois, le roman de Meyrink traite d'un soulèvement populaire alors que Les Nuits fauves racontent un soulèvement ou, plutôt, un dépassement individuel.

16. La chanson du générique ne laisse aucun doute sur la tonalité du film ni sur le rapport entre vie archaïque et vie moderne :

Qui peut dire exactement

Qu'il sait ce qu'est la rage?

Un murmure, un frôlement

Ou la tempête et l'orage?

Juste savoir dire non

A l'appel des sirènes,

Rugir comme un lion

Qui périt dans l'arène.

17. CHEVALIER, Jean, et GHEERBRANT, Alain, Dictionnaire des symboles (Paris : Robert Laffont, 1982), p. 535.

18. BONITZER, Pascal, Peinture et Cinéma. Décadrages (Paris : Cahiers du cinéma/Éditions de l'Etoile, 1985), p. 91. 


\section{RÉSUMÉS}

Cyril Collard, à la fin de sa vie, réalise en apparence une autofiction où apparaissent des éléments de cinéma-vérité bouleversants (Collard mourra peu après la fin du tournage du film). Or, on peut se demander si ce n'est pas les aspects archaïque et fluide de la Vie, pas nécessairement opposés en soi du reste, qui finissent par absorber sa réflexion durant le tournage de l'adaptation de son propre roman. Les Nuits fauves deviennent dès lors une grande tapisserie, à défaut d'être une fresque, où apparaissent des motifs d'une rare beauté. A la débauche que le film mentionne en passant, Collard préfère s'interroger sur l'ébauche d'une vie libérée des faux-semblants, spontanée, ouverte à l'inconnu et soucieuse de défendre un héritage libertaire mais aussi sociologiquement très riche. Ainsi la révolte sexuelle ne serait qu'une condition, une préparation vers un état de conscience appréciant les hommes et les choses à leur juste valeur.

\section{AUTEUR}

\section{THIERRY GIACCARDI}

Docteur ès Lettres et chargé de cours à l'université de Belfast. Ses domaines de recherche sont la littérature francophone et anglophone des $\mathrm{XIX}^{\mathrm{e}}$ et $\mathrm{XX}^{\mathrm{e}}$ siècles, le cinéma (Europe, Amérique) et la civilisation nord-américaine et européenne.

Liste des publications les plus récentes :

In Nouvelles «vues » sur le cinéma québécois:

«Représentation de la chair au théâtre et au cinéma », (numéro 8, hiver 2008).

In Les Débris du sens, Philologicum éditeur :

«Exemples de rédemption dans le cinéma hollywoodien », ouvrage collectif, textes réunis par Pascale Hummel et Frédéric Gabriel, (2008), pp. 283-294.

In Anales de filología francesa, Universidad de Murcia, España :

«Épopée terrestre et épopée céleste : la "base” et le "nom " dans l’Essai sur le principe de Joseph de Maistre », numéro « 1808 », (volumen XVI, 2008), pp. 87-99.

In Revue André Malraux Review, University of Oklahoma, USA :

«L'homme de granit dans Espoir, Sierra de Terruel de Malraux : un essai de métaphysique visuelle ", numéro Malraux et les valeurs spirituelles du XXIe siècle, sous la direction de Michel Lantelme, (volume 36, 2009), pp. 109-120 ;

«La gnose dans Lazare », en préparation.

In La Revue des Lettres Modernes, Série André Malraux, Minard éditeur :

«L'Esthétique du labyrinthe dans La Tête d'obsidienne », numéro Malraux et la question des genres littéraires, sous la direction de Jean-Claude Larrat (volume 13, 2009), pp. 291-307.

In CinémAction :

«Le Train de Granier-Deferre », numéro Le Train au cinéma, sous la direction d'Albert Montagne, (sous presse);

«L'engagement absolu : Une femme à sa fenêtre », numéro Cinéma et engagement : Jorge Semprún scénariste, sous la direction de Jaime Céspedes (sous presse). 